\title{
Orientation Imaging of Nanocrystalline Platinum Films in the TEM
}

\author{
A. Darbal ${ }^{*}$, K. Barmak ${ }^{*}$, T. Nuhfer ${ }^{*}$, D. J. Dingley ${ }^{* *}$, G. Meaden ${ }^{* *}$, J. Michael $^{* * *}$, T.

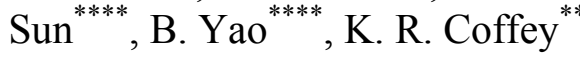 \\ *Department of Materials Science and Engineering, Carnegie Mellon University, \\ 5000 Forbes Avenue, Pittsburgh, PA 15213 \\ ${ }^{* *}$ EBSD Consultants, Salt Lake City, Utah 84105 \\ ${ }^{* * *}$ Sandia National Laboratories, PO Box 5800, Albuquerque, NM 87185-0886 \\ ****Advanced Materials Processing and Analysis Center, University of Central Florida, \\ 4000 Central Florida Boulevard, Orlando FL 32816
}

Orientation mapping based on automated indexing of electron backscatter diffraction (EBSD) patterns in the scanning electron microscope (SEM) is highly successful and relatively simple. However, for nanocrystalline samples, with sub-100 nm grain sizes, the spatial resolution of EBSD is not adequate; hence, it is necessary to use the transmission electron microscope (TEM). In this paper, we report on high-resolution orientation imaging of a nanocrystalline Pt film (Fig. 1a) using the dark-field conical scanning method in the TEM [1]. In this method, a series of dark field images is obtained from the same area of the sample by tilting and rotating the incident beam to form a series of cones. A pixel in the dark field image will become bright when the incident beam has an orientation such that it satisfies the Bragg condition at that point and the diffracted beam passes through the objective aperture. A polar plot of the intensity variation of the same point in each of the dark field images with the incident beam orientation gives a reconstructed diffraction pattern (RDP) (Fig. 1b).

Automated Crystallography for the TEM (ACT) is used to index the RDPs and determine the orientation. In ACT, a mask of predefined size scans the pattern and whenever a number of criteria (relative brightness, slope etc.) are satisfied, a peak (spot) is registered. Using a table of hkl reflections against Bragg angle, the spots are assigned possible miller indices. A series of two-dimensional grids are generated using the found spots and the pattern center to determine the best fit reciprocal lattice plane corresponding to the RDP (Fig. 1c). The orientation of the given point is then established through a voting/ranking scheme, which takes into account any deviation from the base reciprocal lattice plane as indicated by the different intensities of the reflections.

The orientation imaging results for a $50 \mathrm{~nm}$-thick Pt film obtained by analyzing the RDPs is presented. The film was deposited on an oxidized Si (100) substrate and annealed at $800{ }^{\circ} \mathrm{C}$ for $1 \mathrm{~h}$. The choice of $\mathrm{Pt}$ as a high-atomic number element and the annealing conditions were so as to generate a film for which the orientation mapping could be done by EBSD in the SEM as an independent verification of the results obtained from the TEM. Prior to indexing of the RDPs, a grain map is generated based on the position of the brightest spot in the RDP. If the position of the brightest spot for two adjacent points is within a predefined tolerance, then the two points belong to the same grain. The purpose of generating a grain map is to identify the coherent scattering volume and to average the RDPs for points that belong to the same grain. This improves the quality of the RDP and increases the reliability of the indexing procedure. 
Orientation imaging results for two datasets collected from the same Pt sample are presented in Figs. 1d, 1f, 1g and 1h. Results from the analysis of dataset 1 (Figs. 1d and $1 \mathrm{~g})$ show that the Pt film is strongly $<111>$ fiber textured, which is in agreement with the orientation image (Fig. 1e) obtained from the EBSD analysis in the SEM. Selected area diffraction (in the TEM) from the same sample further confirms the presence of strong $<111>$ fiber texture. Results from the analysis of dataset 2 are shown in Figs. 1f and $1 \mathrm{~h}$. Comparison of results from the two data sets shows the sensitivity of this method to the data collection conditions. Absence of a strong $<111>$ texture in Figs. 1f and $1 \mathrm{~h}$ shows that many of the RDPs in dataset 2 are indexed incorrectly. Higher incident beam convergence used in collecting the dark field images for this data set is a possible explanation for the difficulty in accurately indexing the RDPs. It was observed that the indexing solutions for dataset 2 (Figs. 1f and $1 \mathrm{~h}$ ) showed a higher sensitivity to variation in mask size compared to dataset 1 (Figs. $1 \mathrm{~d}$ and $1 \mathrm{~g}$ ).

[1] D. J. Dingley, Michrochim. Acta 155 (2006) 19.
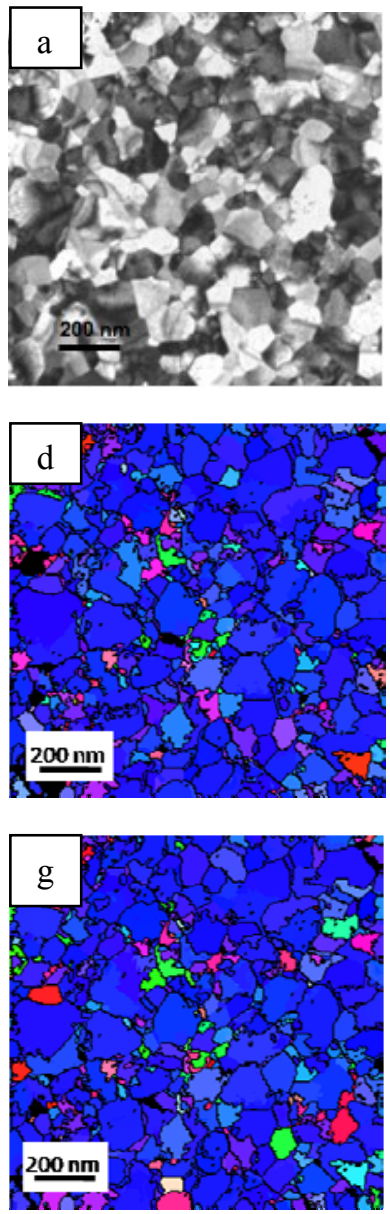
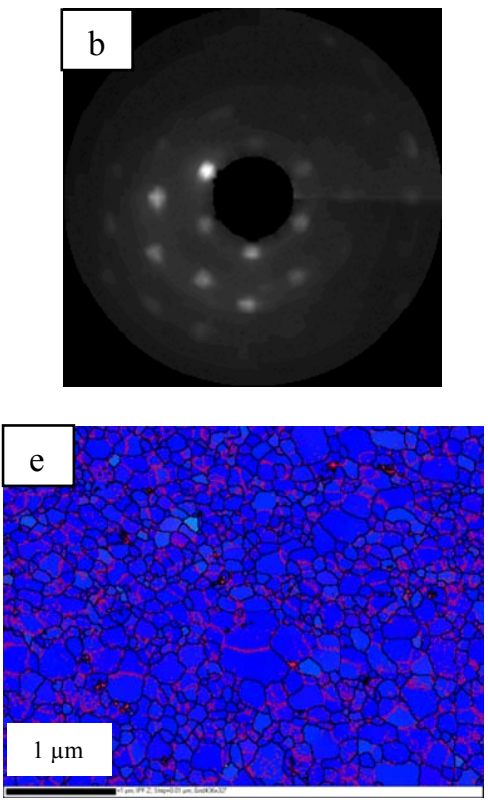

Color key for the IPF map

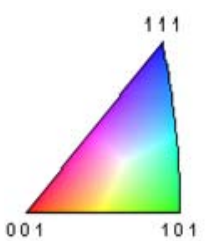

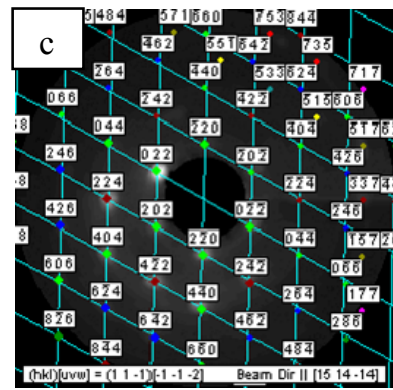
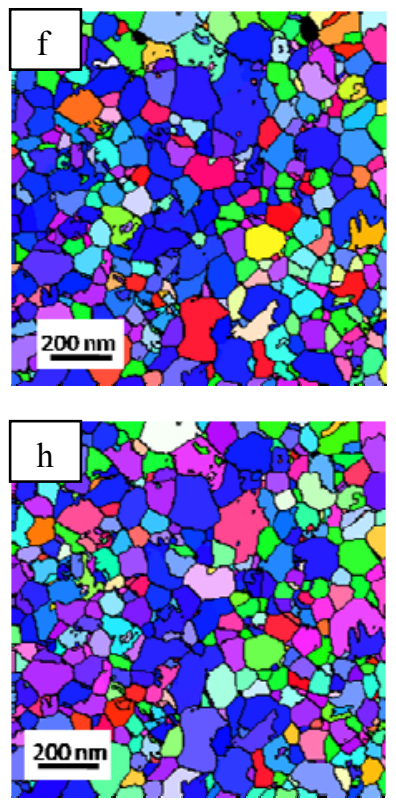

Fig. 1: (a) Bright field image from the $50 \mathrm{~nm}$-thick, annealed Pt film, (b) RDP from a point in the bright field image, (c) RDP indexed using ACT, (d) Inverse pole figure (IPF) map from dataset 1 using mask size 8, (e) IPF map for the same sample using EBSD analysis, (f) IPF map from dataset 2 using mask size 8, (g) IPF map from dataset 1 using mask size 9, (h) IPF map from dataset 2 using mask size 9. 